\title{
Reflective control of attributed entities in feature-based CAD systems using a CARW system manager
}

\author{
F. Mandorli and U. Cugini \\ KAEMaRT Group, Dep. of Industrial Eng., The University of Parma \\ V.le delle Scienze, Parma, ITALY \\ tel. +39-521-905706 - fax. +39-521-905705 \\ e-mail: [ferro, cugini]@labcadl.eng.unipr.it \\ H. E. Otto and F. Kimura \\ Dep. of Precision Machinery Eng., The University of Tokyo \\ Bunkyo-ku, Hongo 7-3-1, Tokyo 113, JAPAN \\ tel. $+81-3-3812-2111-$ fax. $+81-3-3812-8849$ \\ e-mail:[otto,kimura]@cim.pe.u-tokyo.ac.jp
}

\begin{abstract}
Requirements necessary to integrate intelligent computer aided design systems in future KAE/CAM systems aimed at supporting concurrent engineering and the handling of product data, propounding extensions and new developments of system architecture, data structures and functionality. Indispensable among these are an integrated and flexible formalization of the knowledge base and a modular system architecture which can be easily modified to suit current demands. Within our developed framework we propose a homogeneous representation of declarative, procedural, and control knowledge based on a conditional attributed rewriting system (CARW). Design, reasoning and computation are carried out in domains of typed attributed entities, which are replenished with reflective capabilities. Due to the rule-based approach, a high degree of flexibility and modularity can be achieved, while perpetuating the system's overall control in the form of a CARW manager. The objective of this study is to investigate how far our approach satisfies the requirements under consideration. Of major interest are - within the given scope - consistency aspects among attributed entities of different domains and the use of reflective techniques to support status evaluation of design features, their operators as well as design strategies.
\end{abstract}

Keywords

Form-features, rewriting system, attributed entities, reflection, semantics

The engineering design process is investigated and analyzed with results documented in a vast amount of published literature (see Woodson 1966, Yoshikawa 1981, Shigley 1983, Middenford 1996, Tomiyama 1987 for further references) and is concisely defined in SME 1993 as "...Design is an iterative, decision-making activity involving the use of scientific and 
technological information to produce a system, device, or process intended to meet specified needs."

Design decisions involve the form, function and fabrication of parts, components, devices and systems which satisfy a set of specified or implied requirements. Decisions are basically constrained by materials, manufacturing techniques and economics. In mechanical engineering design a significant part of information is represented within or can be related to the form of parts and components.

In what follows, we assume that readers are familiar with feature-based methodologies. In the next two sections we briefly go over some key points about feature methodologies to highlight the problems to be addressed further on.

\subsection{Overview of feature-based methodologies}

In the past decade, a need to improve the capability of CAD systems, to manage not only geometrical information within a stand-alone system, but also to integrate with KAE/CAM systems made research of feature-based methodologies quite popular. The intent of featurebased methodologies is to provide integrated systems with a functionality that allows the user to deal with functional entities of varying complexity. Current literature boasts many definitions of features depending on the context of application (Shah 1988). However, common to all definitions is the inherent concept of a meaningful abstraction of elemental components of a part (pockets, slots, bosses, holes, etc.) that designers and engineers use to evaluate a product. Two main feature-based methodologies have been developed: feature recognition and feature-based modeling.

The intent of feature recognition is to extract from a set of geometrical information representing the object model a set of features that corresponds to a higher conceptual layer than geometry. These are associated to functional meaning within one of the phases involved in the production process. Several techniques exist to extract geometrical information from a $3 \mathrm{D}$ solid representation (Brep or CSG) of the object under analysis. More details and further references are to be found in (Henderson 1994, Kim 1993). As feature recognition can be considered a very sophisticated post-processing of CAD data while feature-based modeling is shifting the problem of functional modeling to the design stage. The intent of the feature-based modeling approach is to provide the designer with a set of functional primitives (design features) to aggregate and modify to build up a complete representation of the designed object. In this work we assume (Pratt 1988, Rossignac 1990, Shah 1991a) that a feature:

- Is a physical constituent of a part.

- Is mapable to a generic shape (realizable or implicit).

- Has engineering significance.

- Has predictable properties.

One consequence of the development of design feature models is the possibility to use high level knowledge incorporated in these models for post-design applications such as optimization of planning and manufacturing or in assembly. For this purpose design features need to be mapped into features that are significant for these contexts. This field of research is usually addressed as feature mapping/transmutation. However, it is not always easy to map such high level knowledge from one context to another without making some reasoning about low level knowledge, i.e., geometry and topology. On the other hand, the distinction between feature recognition and feature mapping methodologies is still rather fuzzy (Shah 1994b). 


\subsection{Motivation and objectives}

The motivation for the presented work is the consideration that, in spite of research on featurebased modeling which has matured to the point that results are being incorporated into commercial CAD systems (Pro/Engineering 1987), some problems still remain open. Features are supposed to embed behavior that matches its user's expectations (Rosen 1993). We assume that a part of the engineering significance of features can be related to a feature's shape, which lead us to a design object representation consisting of different integrated models, residing at different conceptual layers. A feature model allows reasoning about high level (design/engineering) primitives as a boundary model allows a complete geometrical representation of the design object. Managing such an integrated model implies the application of methodologies for the definition of a feature shape with its location and validation. We will refer to existing methodologies for any feature shape and location definition.

Further, we will define validation rules in terms of control of significant geometrical properties and control of significant topological entities of a feature at the instancing stage and during the model evolution. To allow such control a close interfacing between a feature model and a geometrical model is required. The integrated model represents at the object level the declarative knowledge related with a product, i.e., WHAT to do. Allowed operations on the feature model represent the procedural knowledge about a production process, i.e., HOW to do. However, strategies in form of control knowledge about the process also need to be represented, i.e., WHY to do (Nilsson 1982, Diaper 1989).

To realize a flexible and powerful system, both procedural and control knowledge must be highly dynamic. This requires the implementation of reflective functionality within a system. By reflection (Smith 1982, Maes 1987, Maes 1988) we refer to the capability of a system to reason about itself, i.e., to evaluate the current state of computation in terms of both object model evaluation and analysis of performed actions. This evaluation allows a system to reason about the current state of objects and possible actions to be performed next.

We consider a context dependent object model evaluation by incorporating feature recognition / mapping functionality within the system. Analysis of performed actions carried out by means of the introduction of a meta-level model capable of representing feature-based design/recognition/mapping strategies. Within the framework we developed, we propose a homogeneous representation of declarative, procedural and control knowledge, based on conditional attributed rewriting systems (CARW). Design, reasoning, and computation are carried out in domains of typed attributed entities, which are replenished with reflective capabilities, while perpetuating the overall control of the system in the form of a CARW manager.

\section{DEFINITION AND VALIDATION OF FEATURES}

Within our framework (Otto 1992, Mandorli 1993, Otto 1993a) we adopt a multi-layer model to represent design object descriptions. All conceptual layers consist of modeling domains made of sets of related typed attributed entities (cf. section 3.1). Within the scope of this work we will focus on the geometrical layer and the feature layer.

At the feature layer, form feature aspects of the design object are organized in an attributed feature graph, representing functional primitives and their relationships. Particular attributes of feature representations are used to relate conceptual shape aspects to geometrical and topological entities (boundary entities) defining the geometrical appearance of a feature. At the geometrical layer, attributed entities represent the shape of an object in terms of topological and geometrical entities together with their relationships (as in a traditional boundary representation). Further geometrical/topological concepts, e.g., concave or convex edges, and reflective properties (cf. sections 2.1 and 2.2) are also represented and handled. 
a)

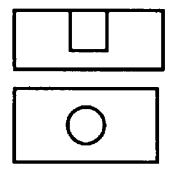

b)

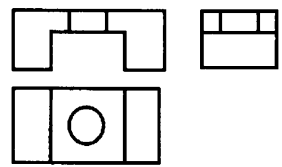

Figure 1 - Feature aggregation without interference (a) and with interference (b)

A feature-based modeling operation usually involves a consecutive revaluation of the model at all layers. A major problem in managing described model structures is how to maintain a correct correspondence between the information represented by typed attributed entities. In particular, if handling form features, consistency among entities of the feature layer and the geometrical layer is relevant. A typical situation, almost certain to induce inconsistency between shape aspects at the feature layer and an actual geometrical representation, is feature interaction.

Let us illustrate this situation within a simple example. Consider a blind hole feature (with given radius and height) positioned on and aggregated with a flange feature (see fig. 1a). Further, assume the hole's nominal shape represented in terms of a complete cylindrical face (lateral face) being open from one side (virtual top face) and closed by a planar face connected at the opposite side (bottom face) as shown in fig. $2 \mathrm{a}$.
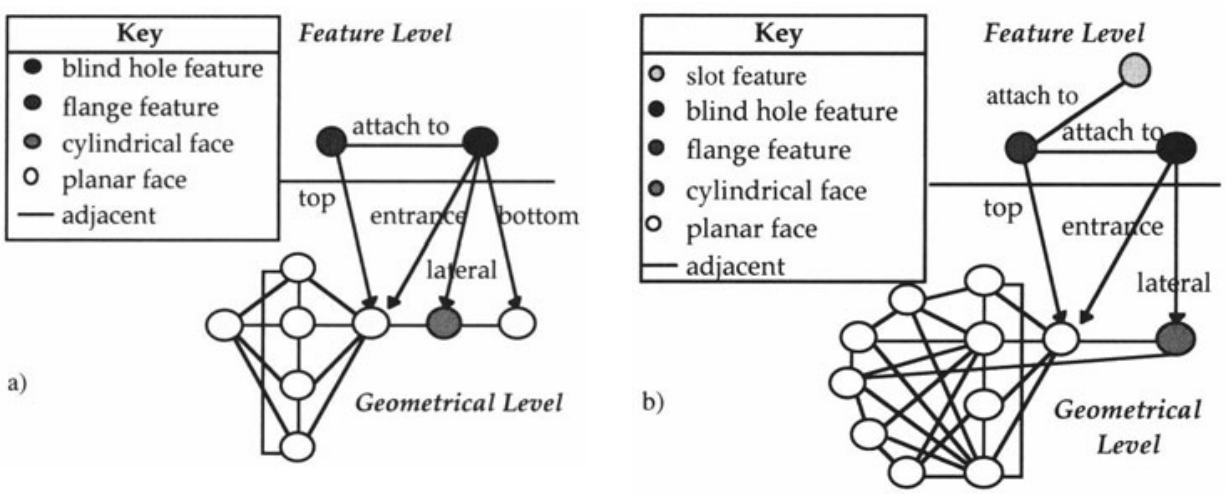

Figure 2 - Topology of a model representation

Within a given context (cf. work process environment Otto 1992, Mandorli 1993) the presence of the hole's bottom face has certain semantics (engineering meaning) that characterize the hole as a blind hole. The insertion of a new feature into the model interacting with the hole and the consequent re-evaluation of the geometrical model may affect the hole's shape in a way that the feature(s) lose(s) associated functional meaning. For example, consider the insertion of a slot feature (cf. fig. 1b) which removes the bottom face of the blind hole, making it a through hole. In consequence, the shape represented by the geometrical model (cf. fig.2b) is inconsistent in respect to the related feature-based representation, where a blind hole feature is still assumed.

Along with feature interaction rise aspects of feature validation, i.e., problems of controlling whether evaluated shape representations (geometrical model) are consistent with their feature-based (functional) description. Currently, we conceptually subdivide, at a macro level, a complex feature-based operation such as aggregate into three functional consecutive actions: 
Instancing:

A new instance of a feature is created by setting a feature's nominal properties, including parameters defining its shape. A Brep of the shape is also created.

Locating:

Methods for feature positioning and a topology definition between the feature and the model must be applied. A boundary evaluation of the model is required to obtain the new geometrical definition of the design object.

Validating:

Because of possible feature interaction, methods for feature validation are applied to ensure that the new feature(s) and those already present in the model are still coherent in respect with their definition.

Feature validation is performed in terms of (reflective) feature attribute control, which includes parameters defining the shape, the location within the feature model and modifications of the topology allowed among boundary entities constituting the feature.

\subsection{Feature definition}

Referring to the previously given feature definition, both shape and engineering significance need to be represented when defining a feature. Note, that a user is interested in the final shape (which might be different from the nominal shape) and the significance of a feature, i.e., the shape and functionality a feature will have after the insertion into the feature model. Techniques that allow to describe a feature's shape in terms of its final shape, using non manifold modelers, and then add it to the model by means of a non manifold Boolean operation can be found in (Ranta 1993). We assume that engineering significance is related to certain properties of a feature's shape. Therefore, particular shapes and their properties need to be preserved after a feature insertion and further maintained during the whole design process. Conforming to above identified conceptual actions involved in a modeling operation we split a feature definition within our framework into three parts:

Definition of primitive shape:

The primitive shape (set during instancing) of a feature is defined in terms of geometrical primitives and parameters that a geometrical modeler (two manifold or non manifold) can use to create the intended shape. Further, an appropriate operation (Boolean for non manifold or two manifold) is specified to add a feature's shape to the model.

Definition of location:

A set of appropriate positioning rules is associated to the feature.

Definition of validation:

A set of rules controlling the geometrical and topological correctness of a feature's shape is associated to the feature.

\subsection{Feature validation}

To validate a feature general control is required for

- limits on dimensions

- compatibility of parent/dependent features

- intersection with other features 
Following our approach given above, the first two validations are covered by our feature definition (see nominal shape and location in section 2.1). To control feature interaction, we propose to apply rules for validation. These rules express a set of properties, that topological and geometrical entities ought to posses if related to a feature. Properties include impositions of the presence as well as absence of selected constituent entities of a feature. For example, consider a blind hole feature where for the bottom a face has to be present, while for the entrance only a virtual face (loop) is appropriate.

Further functionality can be preserved imposing stricter constraints over such entities, e.g., the bottom face should not have internal concave loops while its cylindrical (lateral) face must be bounded by two and only two circular loops. Due to geometrical operations, which include the manipulation of topological/geometrical entities difficulties arise to maintain relationships among entities of different layers (through identifiers within the scope of an integrated geometrical modeler and CARWs).

\subsection{Interfacing features and geometry using an entity monitor}

Problems of identity and extensionality are always present if modeling within formal structures and languages. However, if the identifier scope of a certain modeling/reasoning space is within the scope of one administration / computation facility, as it is the case in most procedural programming languages and term rewriting systems, name clashes and extensionality problems can be solved by renaming and type/term inference. In the approach we have taken, we have no limitation at the implementation level of low level functionality due to our reference kernel architecture (Otto 1992, Mandorli 1993, Otto 1993a), as long as the basic structure of an attributed entity is maintained. Therefore above mentioned issues become a non-trivial problem within the context of geometric modeling based on Breps.

Let us explain the given situation with an example. Suppose we have the definition of a blind hole feature (cf. definition of a fixing hole given in Otto 1994b). Further, to each conceptual topological element constituting the feature's semantics, there is a related topological entity residing at the geometrical layer and being with its geometry a part of a certain shape aspect. Finally, consider the lowest level of implementation administered by a geometric modeling kernel. If we further assume that we have a flat domain of identifiers and a context, defining the scope within one system at the implementation level, we are only able to model consistently a static situation, i.e., relate corresponding entities from the feature layer and the geometrical layer to each other while defining geometry (nominal shape) without any operation (Otto 1995a).

To keep trace of related entities of different conceptual layers, represented and manipulated within different identifier scopes, we need (at the implementation level) an entity monitor. This monitor automatically interprets values of context-bound identifiers and forwards entity related status information. Current values and their annotation of the (object) status domain GMTP_STATE are as following:

- OLD The associated entity is unchanged.

- NEW The associated entity has been replaced by a new entity of the same type.

- SPLIT The associated entity has been split into new entities.

- MERGE The associated entity has been merged with another entity and a new entity has been created.

- DELETE The associated entity has been deleted from the model. 


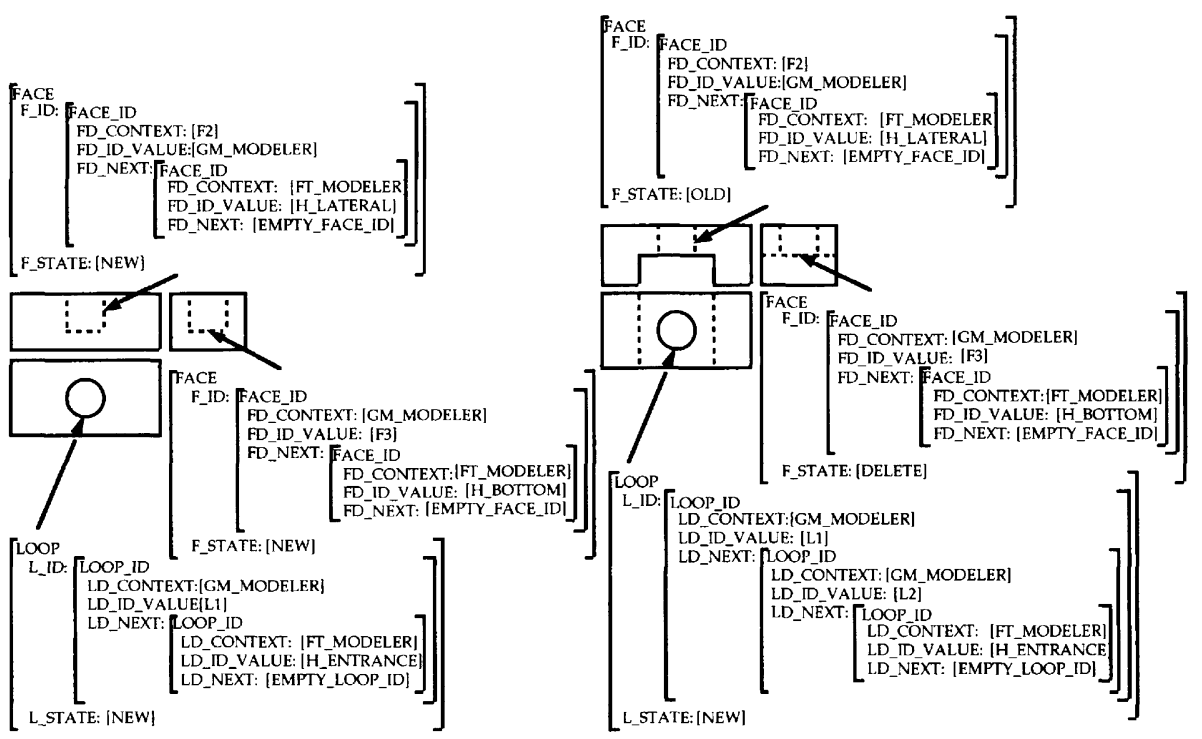

Figure 3 - Evolution of traced identifiers with context among related attributed entities

In fig. 3 the previous example of a hole feature together with a flange feature (see also section 2 and figs. $1 \mathrm{a}$ and $1 \mathrm{~b}$ ), where a slot is finally added, is shown together with their partly evaluated TAE structures to illustrate the entity monitor's tracing activities. Note, due to paper space limitations, only some types of topological entities together with their identifiers and respective context of modelers such as GM_MODELER and FT_MODELER are shown. Their fully evaluated TAE structures, represented in attributed value clusters are given in (Otto 1995b).

\section{A FRAMEWORK BASED ON A CARW MANAGER FOR COMPUTATION}

A key characteristic of design is the iterative aspect of the process based on a continuos decision-making activity that involves reasoning about the present state of the process itself. This results in a continuous improvement as more detailed information becomes available. At each step of the iteration, the design object model is evaluated, to take decisions on the actions to perform next. The model incorporates information about the design object (declarative knowledge) as well as about performed actions (procedural knowledge) and strategies (control knowledge). Therefore, model evaluation involves not only the analysis of a design object, but extends to reasoning about actions and strategies that finally lead to the realization of an artifact (Kimura 1993a,b). Capabilities which involve reasoning about the status of an object or operation, rather than its assigned value or computational result, are known as reflection or self introspection (cf. sections 2.1 and 2.2).

The automation of a reflective activity requires an internal system representation of its status and the capability to take decision on the basis of just this status analysis. The capability of an actor to perform a repetitive action over another actor of the same type is usually called meta (Burattini 1993). Applications of such a concept are mainly found in the theory of meta languages. A meta language is meant to be a language used to represent and perform reasoning about another language. In previous works we have introduced the CARW as a computational 
framework to represent feature-based design objects in terms of (typed) attributed symbols with respective domains. Further feature-based design (Otto 1992, Mandorli 1993, Otto 1993a, Otto 1994a,b) and recognition (Mandorli 1991, Cugini 1992a,b) operations in terms of conditional rewriting rules describe modification/evaluation of the design object model. To provide a reflective capability for our system, we extend the use of a CARW and introduce a meta CARW, which is able to perform actions over a CARW (cf. meta principle of actors above).

Within our framework, we assign to each computational level (object level and meta level in fig.4) one complete and independent CARW to represent not only the design object and appropriate design operations, but design strategies and their handling as well. The new introduced CARW at the meta level, representing control knowledge in terms of status and strategies aims to provide extended reflective computation by means of reasoning not only about the state of a design object, but also about performed actions.

\subsection{Conceptual outline of the computational architecture}

In our work we refer to the general design theory (GDT, Yoshikawa 1981, Tomiyama 1987) as a theoretical framework, to describe design activities. The GDT, as a descriptive model, handles design performance conceptually in terms of knowledge manipulation. A design process can be regarded as a mapping between a function domain and an attribute domain. Both domains, or generally spaces, are defined over the set of an entity concept. The specifications of design solutions can be described within particular entity concepts, i.e., attributes and their topology. For the description of real design process knowledge in GDT, a meta model and the concept of an evolutionary design process model are introduced. A meta model can be regarded as a representation of both the design object and the knowledge about design rules and constraints.

In our framework, entities are formally treated as objects of different domains. Syntax and semantics are determined through properties, which are their attributes (Otto 1993a,b). Attribute values in turn can also be attributed entities of other domains. Entity concepts and attribute domains, as defined in the GDT, can be mapped into schema (cf. conceptual layers) and types with an immanent order of information (Otto 1993b). Those abstract constructs in term can be formally described with typed attributed entity (TAE) structures and semantic models as described in (Otto 1994b,c). Following our approach, we look at features as attributed entities of a particular entity concept. TAE structures can be used to represent features by assigning to each conceptual entity of the form feature/application domain an attribute which has a value of just the type representing this concept. Here design object descriptions are composed of sets of features, each having a particular type assigned.

We have identified a hierarchy of conceptual layers for all used entities represented by TAE structures (Mandorli 1993, Otto 1993a, Otto 1994b). In particular, features (entities of the feature layer) and topological/geometrical elements (entities of the geometrical layer) are mapped into attributed symbols that form the alphabet of our proposed computational framework (fig.4) of a CARW at the object level. Lower level entities are embedded in attributes of higher level entities. These attributes are managed by the semantic part of rules acting on symbols which denote such higher level entities. In (Otto 1994b) we demonstrated how TAE structures can be mapped into sets of attributed symbols to be used as an attributed alphabet of a CARW.

In the next section we will demonstrate how feature-based operations can be mapped into attributed symbols. These symbols in turn define a new alphabet of a meta language, recognized by a meta level CARW. Rules of the meta level CARW represent actions, reasoning over rules of the object level CARW, realizing in this way reflective functionality. In other words, rules at the meta level represent strategies on how to apply feature-based operations (rules of the object level) to the object model. 


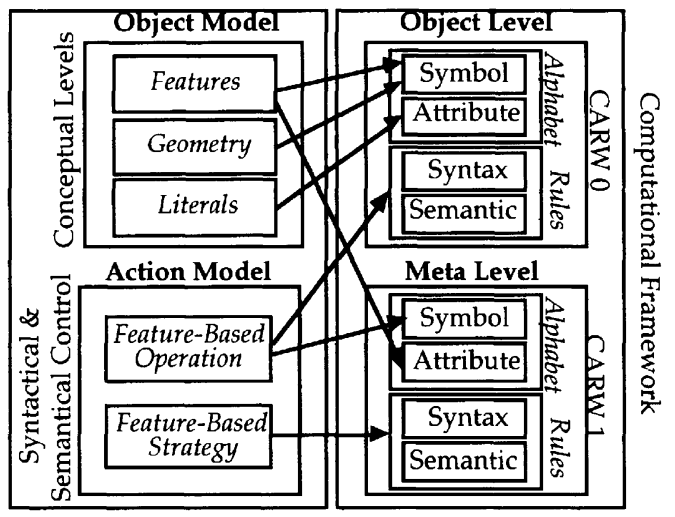

Figure 4 - Relationships between the conceptual and the computational framework

Additionally a CARW manager is defined to perform overall reflective control of a design process by means of the application of appropriate object rules / meta rules within an appropriate object level / meta level CARW. The exclusive use of an CARW formalism to define strategies (meta language) and object/operator descriptions (language) is important, due to the use of one single interpreter for both, generation and execution of strategies. Note, a distinction between the two languages prevents contradictions and paradoxes (Bottoni 1994).

\subsection{Representation of feature-based operations}

We assume that feature-based operations can be characterized by a type, an identifier and attributes that identify structures to which the operation has been applied (TRIAL) and structures to which the operation has been successfully applied (SUCCESS). Let us denote the sets of all used feature-based operations, their types, identifiers, and property characterizing attributes with T, ID, and ATTR, respectively. Now we can recast a feature-based operation definition in terms of entities, consisting of sets (power sets) of a type, attributes and identifiers as follows:

Definition: A description $\Delta$ of a feature-based operation is a triple $\Delta=\left\langle\Delta^{\mathrm{m}}, \Delta^{\mathrm{D}}, \Delta^{\mathrm{P}}\right\rangle$ with $\mathrm{D}_{\mathrm{TYPE}}(\Delta)=\mathrm{T}$, where: $\Delta^{\mathrm{m}} \in \mathrm{Id}$ is the identifier of the described operation, which identifies it uniquely. $\left.\Delta^{D}=\left\{\left\langle a_{1}, v_{1}\right\rangle, \ldots,<a_{s}, v_{s}\right\rangle\right\}, a_{i} \in$ ATTR, $a_{i}-a_{j}$ for $i-j, v_{i} \in D_{a i} i=1, \ldots, s$ is the finite set of the descriptors. TYPE $\in \Delta^{\mathrm{D}}$ denotes the attribute specifying the type of $\Delta ; \Delta^{\mathrm{P}}$ is a collection of sets $\left\{\mathrm{P}_{1}, \ldots, \mathrm{P}_{\mathrm{r}}\right\}, \mathrm{P}_{\mathrm{i}} \in P\left(\mathrm{Id}-\left\{\Delta^{\mathrm{m}}\right\}\right)$ where $P$ denotes the power set and $\left(\cup_{\mathrm{i}=1 \ldots \mathrm{r}}\right.$ $\left.\mathrm{P}_{\mathrm{i}}\right) \cup \Delta^{\mathrm{D}}-\varnothing . \Delta^{\mathrm{P}}$ contains the identifiers of the rules composing the structure identified by $\Delta^{\mathrm{m}}$.

All given representations of an element can be further mapped into a representation of an attributed symbol and consequently a whole object description into a string of attributed symbols. Each symbol consists of an $m+1$ tuple of symbols, starting with its type $t \in T$, a new 
set of attributes including the identifier $\Delta^{\mathrm{Dm}}:=\Delta^{\mathrm{D}} \cup \Delta^{\mathrm{m}}$ and an appropriate coding for the list of composing elements $\Delta^{\mathrm{P}}$. Given an object description in such a form, any data interpretation and knowledge manipulation can be modeled as a rewriting process (Bottoni 1993, Mandorli 1994). Reflection of feature-based operators and strategies, carried out at the meta level, employs values of above mentioned attributes, to reason about the current state of applied operations/strategies during computation.

\subsection{Characteristics and formal definition of a CARW}

Rewriting systems are directed equations used to compute by repeatedly replacing subterms (in a specific context) of a given term with equal terms. Enabling conditions in form of implications together with semantics are given to such systems to specify semantically as well as syntactically when and how rewriting may be performed.

Definition: A CARW is defined by a semantic domain $\mathrm{D}=\left(\Omega_{\mathrm{D}}, \Phi_{\mathrm{D}}, \Gamma_{\mathrm{D}}\right)$ and a rewriting system $\mathrm{RW}=(\mathrm{V}, \mathrm{P}, \mathrm{I})$.

$\Omega_{\mathrm{D}} \quad$ is a set of sets of values.

$\Phi_{\mathrm{D}} \quad$ is a set of functions.

$\Gamma_{\mathrm{D}} \quad$ is a set of predicates, also comprising the constant predicates true and false.

$\mathrm{V}$ is a finite set of symbols called the alphabet.

$\mathrm{P} \quad$ is a finite set of productions.

I is the interpreter, i.e., the specification of the direct generation relation.

$\forall \mathrm{x} \in \mathrm{V}, \exists \mathrm{A}(\mathrm{x})$, called set of attributes, $\forall \alpha \in \mathrm{A}(\mathrm{x}), \exists \mathrm{D}_{\alpha}(\mathrm{x}) \in \Omega_{\mathrm{D}}$ which specifies the possible values an attribute $\alpha$ can assume when associated with the symbol $x . D_{\alpha}(x)$ is called the domain of the attribute $\alpha$ for the symbol $\mathrm{x}$.

Each production $\mathrm{p}$ is of the form:

$<<\omega_{1}, \delta, \omega_{2}>, \varepsilon>$, denoting that for $\delta$ to be rewritten as $\varepsilon$, it must be embedded in the context represented by $\omega_{1}$ and $\omega_{2} . \omega_{1}=\omega_{11}, \ldots, \omega_{1 \mathrm{~m}} \in \mathrm{V}^{*} \omega_{2}=\omega_{21}, \ldots, \omega_{2 \mathrm{n}} \in \mathrm{V}^{*} . \delta \in \mathrm{V}^{+}$is called the rewriting part of the antecedent, $\varepsilon=\varepsilon_{1}, \ldots, \varepsilon_{\mathrm{t}} \in \mathrm{V}^{*}$ is called the consequent.

$\forall p \in F, \exists \gamma_{p} \in \Gamma_{D}, \gamma_{p}: D_{\alpha 1}\left(\omega_{11}\right) \times \ldots \times D_{\alpha s}\left(\omega_{1 m}\right) \times \ldots \times D_{\alpha p}\left(\omega_{2 n}\right) \rightarrow\{$ true, false $\} . \gamma_{p}$ is the condition associated with p.

$\forall \mathrm{p} \in \mathrm{P}, \exists \mathrm{r}_{\mathrm{p}}$ which is a finite set of semantic rules of the form:

$\alpha_{\mathrm{i}}\left(\varepsilon_{\mathrm{j}}\right)=f_{\mathrm{j}}\left(\alpha_{1}\left(\omega_{11}\right), \alpha_{2}\left(\omega_{11}\right), \ldots, \alpha_{\mathrm{s}}\left(\omega_{2 n}\right)\right)$, where $f_{\mathrm{j}} \in \Phi_{\mathrm{D}}$. Semantic rules compute attributes of the consequent as a function of those of the antecedent.

Note, a CARW can be specified as a CAIL (Conditional Attributed Lindenmayer system with Interaction) by means of the use of a parallel interpreter for a simultaneous application of sets of productions (Lindenmayer 1986, Mandorli 1993, Mussio 1988). 


\subsection{The CARW Manager}

The object level CARW is used to represent our integrated design object model and semantics of the set of allowed design/recognition operations.

Definition: The alphabet $\mathbf{V}_{\mathbf{0}}$ of the object level CARW is defined as:

$\mathbf{V}_{\mathbf{0}}=\mathbf{G}_{\mathbf{0}} \cup \mathbf{F}_{\mathbf{0}}$

where:

$\mathbf{G}_{\mathbf{0}}=\{$ topological / geometrical entities $\}$

$\mathbf{F}_{\mathbf{0}}=\{$ defined form features $\}$

A complete definition of the attributes (and related domains) of $\mathbf{G}_{\mathbf{0}}$ together with an example of $\mathbf{F}_{\mathbf{0}}$ can be found in (Otto 1994a,b).

Definition: The set of productions $\mathbf{P}_{\mathbf{0}}$ of the object level CARW is defined as:

$\mathbf{P}_{\mathbf{0}}=\{$ allowed feature-based operations $\}$

Conforming with given CARW rule definitions, each production $\mathrm{p}_{0}^{\mathrm{i}}$ in $\mathbf{P}_{\mathbf{0}}$ consists of a syntactic part, defining symbols (structures) over which an operation is allowed. Further, in the syntactic part of a rule, a set of conditions $\gamma_{\mathrm{p} 0}$ performing semantic control on the domains of the attributes of the symbols is specified.

The meta level CARW is used to represent the knowledge about performed design/recognition activities and allowed design / recognition strategies.

Definition: The alphabet $\mathbf{V}_{\mathbf{M}}$ of the meta level CARW is defined as:

$$
\begin{aligned}
& \mathbf{V}_{\mathbf{M}}=\left\{\mathrm{s}_{\mathbf{i}} \mid \forall \mathrm{s}_{\mathrm{i}} \exists \mathrm{p}_{0}^{\mathrm{i}} \in \mathbf{P}_{\mathbf{0}}\right\} \\
& \forall \mathrm{x} \in \mathbf{V}_{\mathbf{M}} \alpha_{1}=\text { TRIAL, } \alpha_{2}=\text { SUCCESS }
\end{aligned}
$$

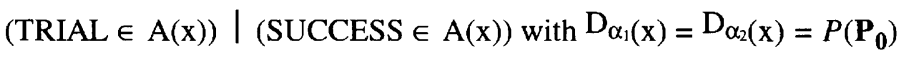

Definition: The set of productions $\mathbf{P}_{\mathbf{M}}$ of the meta level CARW is:

$\mathbf{P}_{\mathbf{M}}=\{$ allowed feature-based strategies $\}$

Each $\mathrm{p}_{\mathrm{M}}^{\mathrm{i}}$ in $\mathbf{P}_{\mathbf{M}}$ is defined by a syntactic part, where the antecedent specifies the (sub)state of the interpretation process in which the meta rule (strategy) can be applied. The consequent specifies the string of symbols, corresponding to the set of names of (meta) rules to be applied in the following step of the computation.

The semantic part of rules incorporates reflective control by means of a set of conditions imposed on the attributes to reason about previously applied rules which are within the syntactic part. To allow the integrated management of attributed elements at the computational level sequences of attributed symbols representing a design object description and operators/strategies are administered within a state model (fig.5). Each state of computation, 
supervised by the CARW manager and executed by an interpreter of the object and meta level, respectively, is represented as a transition in the state model, while computing appropriate actions to be performed in further steps.

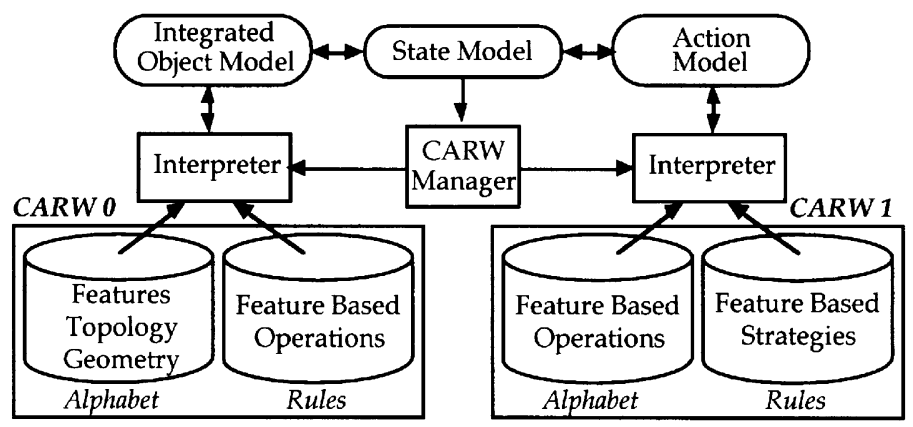

Figure 5 - CARW manager and state model

Definition: The state $\mathrm{s}$ of computation $\mathrm{C}$ at step $\mathrm{k}$ is defined within our state model $\mathbf{S}(\mathrm{k})$ as

$\mathrm{S}(\mathrm{k})=$ OBJECT $_{0}(\mathrm{k}) \circ \operatorname{ACTION}_{\operatorname{COM}}(\mathrm{k}) \circ \operatorname{ACTION}_{\text {NEXT }}(\mathrm{k})$

where:

- is the concatenation of symbols

OBJECT $_{\mathbf{0}}(\mathrm{k})=\left\{\mathrm{x}_{0}^{\mathrm{i}} \mid \forall \mathrm{k} \in \mathbf{N}^{+} \mathrm{x}_{0}^{\mathrm{i}} \in \mathrm{V}_{\mathbf{0}}\right\}$ consists of a set of elements, denoting the integrated object model (string of CARW 0)

$\operatorname{ACTION}_{\operatorname{CoM}}(\mathrm{k})=L\left(\mathrm{x}_{\mathrm{i}} \mid \forall \mathrm{k} \in \mathbf{N}^{+} \forall \mathrm{x}_{\mathrm{i}}\left(\exists \mathrm{p}_{0}^{\mathrm{i}} \in \mathbf{P}_{\mathbf{0}}\right) \vee\left(\exists \mathrm{p}_{\mathbf{M}}^{\mathrm{i}} \in \mathbf{P}_{\mathbf{M}}\right)\right)$ represents a list of actions, already completed (with or without success) to obtain $\mathbf{O B J E C T}_{\mathbf{0}}(\mathbf{k})$

$\operatorname{ACTION}_{\text {NeXT }}(\mathrm{k})=L\left(\mathrm{x}_{\mathrm{i}} \mid \forall \mathrm{k} \in \mathbf{N}^{+} \forall \mathrm{x}_{\mathrm{i}}\left(\exists \mathrm{p}_{0}^{\mathrm{i}} \in \mathbf{P}_{\mathbf{0}}\right) \vee\left(\exists \mathrm{p}_{\mathrm{M}}^{\mathrm{i}} \in \mathbf{P}_{\mathbf{M}}\right)\right)$ denotes a list of actions, suggested for performance in future steps

Note, in given context above it is important to handle lists instead of sets due to the correlation between operators/actions and states of computation (aspect of sequential order).

The manager $\mathrm{M}$ manages rules and meta rules, using given CARW notation and the state model $\mathbf{S}(\mathrm{k})$ as follows:

1 reading the first symbol $\mathbf{s}_{1}$ contained in $\operatorname{ACTION}_{\mathbf{N E X T}}(\mathrm{k})$, adding it to $\operatorname{ACTION}_{\mathbf{C O M}}(\mathrm{k})$ and erasing it from ACTION NEXT $(k)$.

2 executing the set of (meta)rules specified by the symbol $\mathbf{s}_{1}$, thus modifying either OBJECT $_{0}(k)$ (if $s_{1}$ is the name of an object rule) or $\operatorname{ACTION}_{\mathbf{N E X T}}(\mathrm{k})$ (if $\mathbf{s}_{1}$ is the name of a meta rule).

3 updating attributes TRIAL and SUCCESS of $\mathbf{s}_{1}$ in $\operatorname{ACTION}_{\mathbf{C O M}}(\mathrm{k})$ 
To illustrate the approach taken, some annotated design steps with their related knowledge handling are demonstrated on a real test part. The example, a hinge bracket, taken from results of the FEMOD project (Bozza 1992, Bozza 1993), is related to the aeronautical context of milled parts manufacturing. Due to paper space limitations, only a selection of the entire test data is discussed, sufficient to demonstrate how information is mapped and processed in case of a real application. The data were used to verify theories and concepts developed within the framework we introduced. Of main interest, besides consistency aspects of feature semantics, is the investigation of reflective functionality integrated in our proposed framework and used at both the object and the meta level.

As an implementation environment for data representation, domain modeling and computation, several different modules of a prototype system developed following our reference kernel architecture (Otto 1992, Mandorli 1993, Otto 1993a) have been used.

\subsection{Informal design object and design rules specifications}

An airframe is composed of a structure, the skeleton of the aircraft and of a covering, the external skin that determines the aircraft contour. The milled parts are intrinsically resistant so they are located mainly in the airframe structure. Milled parts are manufactured using milling machines and lathes that remove particles of metal (chips) from an initial metal block or an unfinished product obtained by die casting. The example of a milled part considered, is a hinge bracket shown in fig.6.

The functionality of a hinge bracket is to support the joint of the movable mechanism of the hinge. Hinge brackets are positioned on the rear spar of a wing to support flaps. Main constraints in designing a hinge bracket are the position and the diameter of the hinge hole as well as positions and diameters of the fixing hole present on the wing spare. The shape of the hinge bracket is made by means of spatially surrounding such holes. We can identify following features, composing the hinge bracket:

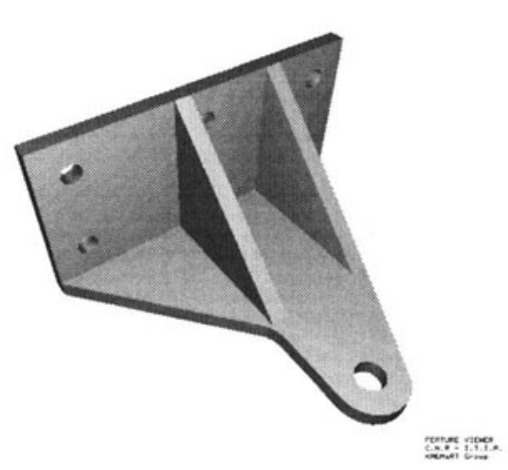

Figure 6 - Hinge bracket

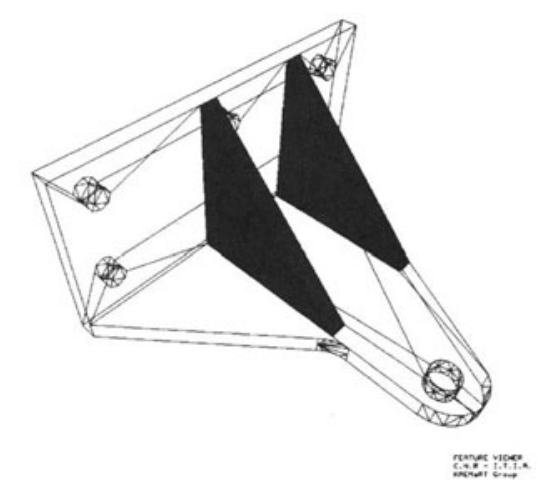

Figure 7 - The two stiffening flanges on the hinge bracket

\section{Fixing flange:}

Used to support fixing holes. Its shape and dimensions are constrained by the number and position of the fixing holes and the environment. 
Fixing holes:

Used to fix the hinge bracket to the wing spare. Diameters and positions are constrained by fixing holes, present on the wing spare.

\section{Hinge lug:}

Used to support the hinge hole. Its shape is constrained by the position and dimension of the hinge hole and the environment.

\section{Hinge hole:}

Used to support the hinge joint. Its dimension is selected from standards.

\section{Stiffening flanges:}

Used to stiffen the part. Shape and dimensions are selected from standards to minimize the weight for a bending load stiffener.

The shape of the fixing flange is first designed by taking into account positions of the environment, where the hinge will be placed (fixing holes, spare dimensions). The hinge bracket design proceeds step by step, determining the geometry of the previously listed features. The fixing flanges geometry (fig.8) is of rectangular shape, with dimensions constrained by the two following rules:

\section{Flange outline:}

The upper and lower outline of the flange requires a tolerance of $2 \mathrm{~mm}$ in regards to the environment to enable assembly.

\section{Flange width:}

The width $\mathrm{W}$ of the flange is calculated as $\mathrm{W}=\mathrm{D}+2 *(2 * \mathrm{~F} 0)$

where $\mathrm{D}$ is the maximum distance between fixing holes and F0 is the fixing holes' diameter.

a)

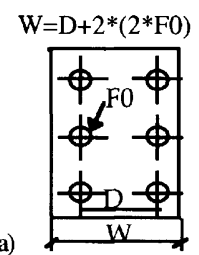

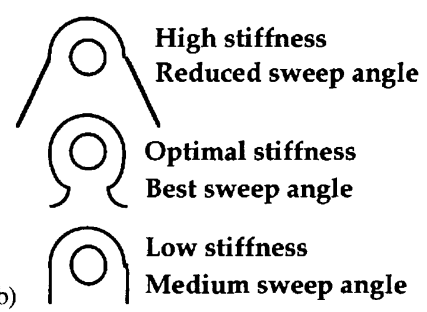

b)

Medium sweep angle

Figure 8 - Flange related to fixing holes and Stiffness and sweep angles

Fixing holes are then positioned on the fixing flange. Positions and diameters are selected on the base of positions and diameters of holes present on the wing spare. The hinge lug shape can be selected from a set of standard shapes. The selection is made considering stiffening properties of the part and a sweep angle, allowed for the joint motion (fig.8b). Dimensions and location of the lug are designed to provide support for the hinge hole. Rules exist that relate the hole diminution and the lug dimension. The basic geometry can then be modified because of constraints imposed by the environment. 
a)

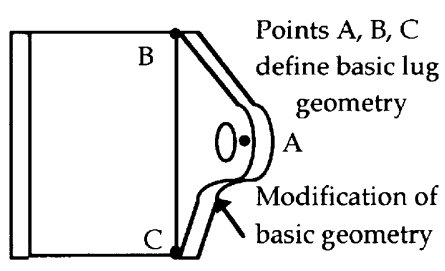

b)

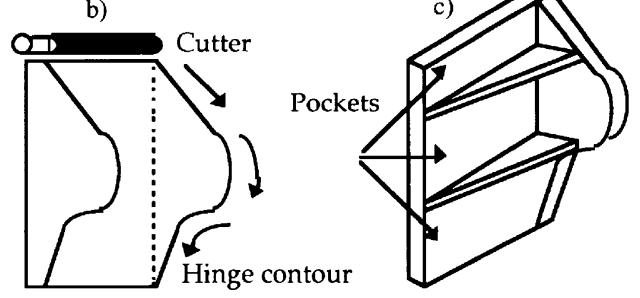

Figure 9 - Basic geometry of a hinge bracket

Finally, stiffening flanges are added to stiffen the part and support the calculated load. Stiffening flanges are selected from standard shapes to minimize the weight for a bending load stiffener. Stiffening flanges are positioned between the fixing flange and the hinge lug. Positioning must be done carefully, to avoid intersection with fixing holes, the hinge hole and forbidden areas on the lug. Once a hinge bracket has been designed, the model is analyzed to identify the appropriate machining process. At this stage, the manufacturing expert looks for machining features, present on the part to make further decisions based on them. In some cases, machining feature can be directly derived from the feature based representation of the model (feature mapping). Referring to our example, the raw material is first machined to obtain the outer contour of the hinge bracket (fig.9b), which corresponds to the outer contour of the hinge lug.

However, in many cases, feature mapping from the design context to the manufacturing context is not a trivial problem. In our chosen example, the next machining step is the contouring of the stiffening flanges. From the manufacturing point of view, significant features are the three separate pockets, as shown in fig.9c. Those pockets are not explicitly present in the feature-based representation of the model. Nor do they exist as a direct mapping (stiffening flanges / pockets). In such cases, we propose to adopt feature recognition, i.e., analyze given geometry and try to relate features of interest.

\subsection{The implemented prototype test environment}

A prototype version of our framework has been implemented and extended to validate our proposed approach and to test reflective functionality on a real test part. The implemented prototype environment consists of several different modules. A geometric modeler, an entity monitor, a feature modeler based on a CARW interpreter and a CARW manager.

We have adopted ACIS (ACIS 1994) as a geometric modeling kernel due its support of an open system architecture based on libraries of functions and its capability to handle attributed entities, essential for the integration with our reference kernel architecture. The ACIS 3D toolkit has been used to specify and realize our modules and to maintain a tight interfacing with the geometric kernel. Programming languages supported by the toolkit are Scheme and C++. We have implemented the monitor using $\mathrm{C}++$, while the $\mathrm{CARW}$ interpreter and the manager are specified within Scheme.

\subsection{Example of reflective control}

In this section we illustrate how reflection within the object model as well as the action model is used to maintain feature semantics. As an example, we will discuss previously introduced hinge 
bracket, implemented within our test environment and related to the contexts of design and manufacturing.

Form features are implemented as typed attributed symbols containing information about a feature's type, shape, location and validation. Shape information contains dimensions as well as geometric modeling operations, to be sent to a geometric modeler kernel to realize the shape of a feature, and add it to the current model (by means of performing a Boolean union or subtraction). A feature's shape can be simple, as in case of the previously mentioned fixing flange, stiffening flanges or holes. There can be described by using basic modeling operations, creating traditional 3D primitives such as rectangular block, triangular block and cylinder. More complex shapes, as in case of the hinge lug, can be described using operations to create a swept profile. A feature's location allows positioning in terms of relating reference systems, belonging to a feature's faces. Features are positioned through appropriate rotation and translation operations, executed at the geometrical layer. Referring to our example, stiffening flanges are located on the hinge by positioning the bottom face of the stiffening flanges flush with the superior face of the fixing flange. The back faces of the stiffening flanges are positioned flush with the front face of the hinge lug, while the left faces of stiffening flanges are positioned considering a fixed distance from the left face of the fixing flange.

Feature validation is performed by taking advantage of reflective functionality, provided by the entity monitor. If a boundary evaluation of a related feature model is performed by means of applying geometric modeling operations, the monitor realizes (object) reflection by tracing all relevant boundary entities and updating their STATE attribute. Let us now explain in detail how feature consistency can be controlled within our framework by means of coupling validation rules and reflective functionality. Referring to our example, the intent is to maintain the functional meaning of fixing and hinge holes, already present in the model, when stiffening flanges are added to the hinge bracket. Spatial interference among the holes/flanges is supposed to be detected without inserting further explicit topological relationships between the holes and the stiffening flanges (cf. section 1.1). When a new instance of a fixing hole is created, its shape (a cylinder) is created by a geometric modeler in form of a boundary representation. The external loops of the two planar faces of the cylinder are related to attributes (cf. ENTRANCE_1, ENTRANCE_2 in Otto 1994a,b) at the feature layer. These attributes take only values in a certain domain, i.e., they are linked to geometrical semantics of a feature and therefore require control in form of associated validation rules. In this case, validation results in testing, whether those loops are external, cylindrical or convex.

After instancing, the feature is positioned and inserted in the current model with a new node in the attributed feature graph, a subtracted cylinder in the Brep, and its STATE attribute set to NEW. Following a geometrical evaluation of relevant topological/geometrical entities newly created identifiers and modeler contexts are recorded, while new values to STATE attributes are assigned. Note, due to the reflective attribute STATE, only modified entities (with attribute values different from OLD) need to be validated. Each new feature insertion causes a further evaluation of the geometrical model including a repeated activation of the monitor. If a newly inserted feature is not interfering with any other features, already present in the model, the STATE attribute of all topological / geometrical entities remains OLD and no validation rules are executed. However, if interference among features occurs, in our example between already present fixing holes of the fixing flange and a new inserted stiffening flange (fig. 10a), the redefined geometrical model has to be validated. In this case, the new split loop (not its opposite old loop, which still has its STATE attribute value OLD) of one fixing hole has to be validated again, which results in the detection of entirely or partly covered features.

Within our framework, feature-based operations are described in terms of rewriting rules. Rewriting rules represent sets of actions used to perform both feature-based design and feature recognition/transmutation. Feature-based design comprises feature instancing, feature location, feature validation and boundary evaluation. 


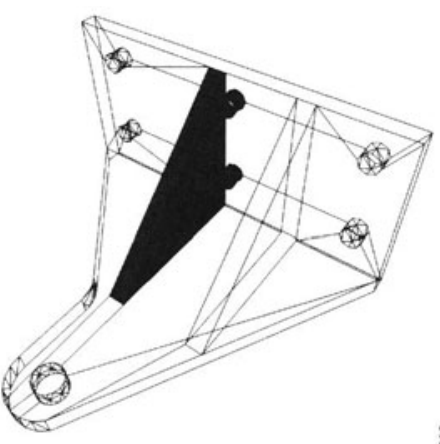

a)

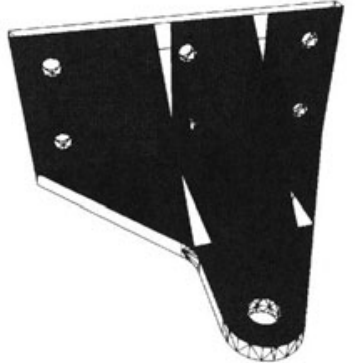

b)

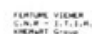

Figure 10 - Stiffening flange intersecting with two fixing holes and three recognized pockets

Feature recognition rules have been implemented to map features belonging to different contexts such as manufacturing, assembling, grasping, etc., by examining design features already present in the model, as well as context related knowledge. Referring to our example, the object level CARW is used to identify the three pockets on the hinge bracket by means of using appropriate rules for machining feature recognition. Fig $10 \mathrm{~b}$ shows the set of faces belonging to the pockets to be manufactured by milling operations.

Our application of conditional attributed rules for feature recognition is a further example of data driven reflection. The antecedent part of a feature recognition rule searches the geometrical model for the set of topological/geometrical entities, enabling possible candidates to be recognized as a feature. Next, a set of conditions is applied over the attributes of the selected entities to check if the topology of entities and their geometrical properties correspond to those of the feature, the rule is supposed to recognize. If all conditions are satisfied, the recognized feature is inserted in the model. In the case of the hinge's pockets, one rule is used to recognize the central pocket, while another rule is used to recognize the lateral pockets. In the case of the rule for recognizing the central pocket, the antecedent $\delta$ selects a set of entities made of four planar faces bounded by at least three consecutive concave edges with a solid angle value equal to $270^{\circ}$. The condition $\gamma_{p}$ is testing, if those four faces are connected by those concave edges. The recognition rule for lateral pockets has an antecedent $\delta$, selecting a set of entities made by three planar faces bounded by at least two consecutive concave edges with a solid angle value equal to $270^{\circ}$. The condition $\gamma_{\mathrm{p}}$ is testing, if these three faces are connected by identified concave edges. Note, the application of the second rule to recognize the two lateral pockets, also yields a twice recognized central pocket. To avoid such recognition anomalies by applying rules in a proper order and to reject redundant recognized features within one context, meta control is required to form strategies.

Rewriting rules at the meta level CARW represent meta rules, reasoning about object level rules. Those meta rules embed reflective functionality by means of evaluating information located in rules of the object level. The interpreter of the meta level CARW has been realized as an extension of the interpreter of the object level CARW, to realize processing of meta level rules (meta rules). Meta rules are used to represent strategies on how to apply feature-based design and recognition operations. These meta rules use extended operator/strategy reflection to realize specified design and recognition activities. Sets of recognition rules to be applied are selected on the base of the previously performed actions and their results. 
Referring to the hinge example, one possible strategy to control the application of certain recognition rules (e.g. the recognition of pockets), requires as a precondition the presence of two stiffening flanges in the model (fig.7). If no stiffening flanges are present, there is no need to start the recognition of pockets. This strategy (meta rule) can be represented within our meta level CARW as a rule, whose antecedent $\delta$ is searching in the action model for symbols representing the activity of adding two stiffening flanges to the model and whose set of conditions is testing whether these actions have been successfully completed, i.e., relevant entities representing rules of the object level have an entry in the attribute SUCCESS. If all conditions are fulfilled, the set of actions describing this particular recognition of pockets is added to the set of proposed actions (cf. ACTION NEXT $(\mathrm{k}$ ) in section 3.6), to be performed next. This set includes the rule for lateral pocket recognition, the rule for central pocket recognition and the rule that rejects redundant recognized features.

\section{CONCLUSION}

The use of reflection within the object model and the action model has been demonstrated on our implemented test part, the hinge bracket, considering design and manufacturing as given contexts. In particular, the application of object model reflection to detect semantic inconsistencies such as open features becoming closed, closed features becoming open and in general spatial interference, have been detected by execution of associated validation rules. Cases where relevant modified entities of the geometrical model failed at least once need to be further examined.

Feature interactions which result in a deleted feature due to addition/subtraction of a larger feature covering the former, have not been discussed within the given example. However, they can be detected by inspecting the set of relevant topological/geometrical entities involved in a geometrical operation, induced by a feature-based operation at the feature layer, while comparing all entities with a STATE attribute value DELETE to those composing the feature's shape in the Brep.

Reflection within the action model has been discussed in the context of manufacturing and the recognition of manufacturing features related to the process of milling. The non-trivial example, to compose a strategy out of given information about performed actions, their status, and a feature-based model for recognizing a set of pockets, showed how essential reflective methodologies are. Without the information on the performance of previously carried out actions it would be rather difficult to compose feature-based strategies, as demonstrated in the example. Additionally, aspects of feature instancing and feature positioning have been integrated in our experiments to attain consistent feature handling.

Reflection is important not only from the conceptual point of view, but has proved to be a fundamental part (together) with the context-dependent identifier in the implementation of an integrated model implementation. Although, the degree of achieved integration showed promising results, it is still a field which needs further improvements, theoretically as well as practically.

With current implementation of our prototype test environment, feature-based operations, which produce inconsistencies in the model (cf. concept of safe/unsafe operators in (Otto 1992, Mandorli 1993), i.e., fail to succeed, are rejected. In further extensions, we would like to combine the mentioned operator concept with methodologies for a user-driven redefinition and revaluation of modified features. 


\section{ACKNOWLEDGEMENTS}

We would like to thank Cesare Zecca for participating in further improvements and extensions of our implemented prototype environment, especially the entity monitor.

\section{REFERENCES:}

ACIS (1994) 3D Toolkit: Extension Reference, Spatial Technology, Inc., Boulder, Colorado.

Bottoni, P., Mussio P. and Protti, M. (1993) A data structure for form feature based image interpretation, in: Proceedings of 7 th Int. Conf. on Image Analysis and Processing.

Bottoni, P., Cugini, U., Mussio, P., Papetti, C. and Protti (1994) M., A System for Form Feature Based Interpretation of Technical Drawings, in: Machine Vision and Applications, Special Issue: Machine Vision and Image Processing Applications in Italy.

Bozza, I., Colombo, G., Cugini, C., Vicini, I., Coquebert, E. and Feru, F. (1992) Knowledge extraction of sheet metal and milled parts on design and manufacture, Subtask 1.4.1, Final Report, BRITE project FEMOD BREU-0333 C, Milano.

Bozza, I., Cugini, U., Moroni, F. and Vicini, I. (1993) Extention of know-how extraction, Subtask 1.4, Final Report, BRITE project FEMOD BREU-0333 C, Milano.

Burattini, E., Cordeschi, R., Criscuolo, G., Minicozzi, E., Tamburrini, G. and Trautteur, G. (1993) Reflection, Self-Reference and Introspection under the Aegis of Meta, Progetto Finalizzato Robotica, Sottoprogetto Governo dei Robot, Draft Report, in Italian, I.T.I.A. - C.N.R., Milan.

Chung, J.C.H. and Schussel, M.D. (1990) Technical Evaluation of Variational and Parametric Design, Computers in Engineering, V. 1, pp. $289-298$.

Cugini, U., Mandorli, F. and Vicini I. (1992a) Form Features Recognition as a Technique for CAD-CAM Integration, in: Proceeding MICAD '92, pp. 115-126, Paris.

Cugini, U., Mandorli, F. and Vicini, I. (1992b) Using Features as Knowledge Formalization for Simultaneous Engineering, in: Proceedings of the IFIP TC5/WG5.3 Eight International PROLAMAT Conference, Man in CIM, G.J. Olling and F. Kimura (eds.), pp. 337-349, Tokyo.

Diaper, D. (1989) Knowledge Elicitation: principles, techniques and applications, Ellis Horwood series in expert systems, Ellis Holwood limited, Chichester.

Henderson, M.R., Srinath, G., Stage, R., Walker, K. and Regli W. (1994) Boundary Representation-based Feature Identification, Advances in feature based manufacturing, Elsevier Science B.V.

Kimura, F. (1993a) Product and Process Modelling as a Kernel for Virtual Manufacturing Environment, Annals of the CIRP, 42/1, pp. 147-151.

Kimura, F. (1993b) A Computer-supported Framework for Concurrent Engineering, in: DIISM'93, Preprints M of JSPE-IFIP WG 5.3 Workshop on The Design of Information Infrastructure Systems for Manufacturing, Tokyo, Japan.

Kim, Y.S. (1993) Volumetric Feature Recognition Using Convex Decomposition, Advances in feature based manufacturing, Elsevier Science B.V., 1994.

Laakko, T.(1994) Incremental Feature Modeling: Methodology for Integrating Features and Solid Models, Ph.D. Thesis, Acta Polytechnica Scandinavica, Mathematics and Computer Science Series, 63.

Lindenmayer, A.( 1986) Mathematical models for cellular interaction in development I - II, Theoretical Biol., 18 , pp. $280-315$.

Maes, P. (1987) Concepts and Experiments in Computational Reflection, in: OOPSLA 87, Conference Proceedings, pp. 147-155.

Maes, P. (1988) Computational Reflection, Knowledge Engineering Review, 3, pp. 3-19.

Mandorli, F. and Vicini, I. (1991) A form feature automatic recognition system, in: Proceeding ICAR'91, Pisa, pp.1398-1403.

Mandorli, F., Otto, H.E. and Kimura, F. (1993) A reference kernel model for feature-based CAD systems supported by conditional attributed rewrite systems, in: Second ACM/IEEE symposium on solid modeling and applications - Solid Modeling '93, pp. 343 - 354.

Mandorli, F. (1994) Interactive Knowledge Elicitation For Application Dependent Feature Evaluation Supported By Conditional Attributed Rewriting Systems, Report, Dept. of Industrial Engineering, University of Parma. 
Middendorf, W.H. (1996) Design of Devices and Systems, Marcel Dekker Inc., New York.

Mussio, P., Padula, M. and Protti, M. (1988) Attributed conditional L-systems: a tool for image description, in: Proceeding of 9th International Joint Conference on Pattern Recognition, IEEE Computer Society Press, pp. 607-609.

Nilsson, N.J. (1982) Principles of Artificial Intelligence, Springer-Verlag Ed., Berlin, Heidelberg, New York.

Otto, H.E. and Mandorli, F. (1992) A Reference Kernel for Feature-Based CAD-Systems; Theoretical and Practical Aspects, Research Note RN-KLO-7-92, Dept. of Precision Machinery Engineering, University of Tokyo.

Otto, H.E. and Kimura, F. (1993a) Integrated Entity Concepts and Attributed Structures as a Base for Feature Modeling, in: Proceedings of JSPE Symposium on Factory Automation and Computer-Integrated Manufacturing, Kyoto.

Otto, H.E. (1993b) On mathematical domain structures and typed attributed features: Some thoughts and experiments, Research Note RN-KLO-1-93, Dept. of Precision Machinery Engineering, University of Tokyo.

Otto, H.E. and Kimura, F. (1994a) Denotational Spaces for Geometrical Semantics of Design Form Features Based on Typed Attributed Entity Structures, in: International Conference on Computer Aided Geometric Design, Penang.

Otto, H.E., Mandorli, F., Cugini, U. and Kimura, F. (1994b) Domain-Oriented Semantics For Feature Modeling Based On TAE Structures Using Conditional Attributed Rewriting Systems, in: ASME International Computers in Engineering Conference, Minneapolis, pp. 13 - 27.

Otto, H.E. and Kimura, F. (1995a) Feature Modeling Based on Attributed Entities and Appropriate Mathematical Domains, International Journal Robotics and Computer-Integrated Manufacturing, to appear.

Otto, H.E. (1995b) Identity and Extensionality of Typed Attributed Entity Structures within Feature-Based CADSystems, Research Note RN-KLO-1-95, Dept. of Precision Machinery Engineering, University of Tokyo.

Pratt, M.J. (1988) Synthesis Of An Optimal Approach To Form Feature Modeling, in: Proceeding of ASME Computer and Engineering Conference, San Francisco, California, pp. 263-274.

Pro/Engineer, (1987) Introductionary guide: Concepts and capabilities, Parametric Technology Corporation, Waltham, Massachusetts.

Ranta, M., Inui, M., Kimura, F., Mäntylä, M. (1993) Cut and Paste Based Modeling with Boundary Features, in: Second Symposium on Solid Modeling and Applications, J. Rossignac, J. Turner, and G. Allen (eds.), Montreal, Canada, pp. 303-312.

Rossignac, J.R. (1990) Issues On Feature-Based Editing And Interrogation Of Solid Models, Computers \& Graphics, pp. 149-172.

Rosen, D.W. (1993) Feature-Based Design: Four Hypotheses for Future CAD Systems, Research in Engineering Design, 5, 3 \& 4, pp. 125 - 139.

Shah, J.J., et al. (1988) Current Status of Features Technology, Report \#R-88-04.1, CAM-I, Arlington, Texas.

Shah J.J. (1991a) Assessment of features technology, Computer-Aided Design, 23, 5, pp. 331 - 343.

Shah J.J. (1991b) Conceptual development of form features and feature modelers, Research in Engineering Design, N. 2, pp. $93-108$.

Shah J.J., Ali, A. and Rogers M.T. (1994a) Investigation of Declarative Feature Modeling, in: Proceedings of the ASME '94 Computers in Engineering, V. 1, pp. 1 - 11.

Shah J.J., Mantyla M. and Nau, D. (1994b) Introduction to Feature Based Manufacturing, Advances in feature based manufacturing, Elsevier Science B.V.

Shigley, J. and Mitchell, L. (1983) Mechanical Engineering Design, McGraw-Hill, New York.

SME, (1993) Society of Manufacturing Engineers, Tool and Manufacturing Engineers Handbook, V. 5, Manufacturing Management, Ch 13, Design for Manufacture, R.F.Veilleux and L.W. Petro (eds.) fourth edition.

Smith, B. C. (1982) Reflection and Semantics in a Procedural Language, Ph. D. Thesis, Massachusetts Institute of Technology, Cambridge, Massachusetts.

Tomiyama, T. and Yoshikawa, H. (1987) Extended General Design Theory, in: Design Theory for CAD Proceedings of the IFIP Working Group 5.2 Working Conference, H. Yoshikawa and E.A. Warman (eds.), pp. 95-124.

Woodson, T.T. (1966) Introduction to Engineering Design, McGraw-Hill Book Co., New York.

Yoshikawa, H. (1981) General Design Theory and a CAD System, in: Man-Machine Communication in CAD/CAM - Proceedings of the IFIP Working Group 5.2-5.3 Working Conference, T. Sata and E. A. Warman (eds.), pp. 35-53. 